\title{
MICRO-MECHANISMS OF CONCRETE FAILURE UNDER CYCLIC COMPRESSION: X-RAY TOMOGRAPHIC IN-SITU OBSERVATIONS
}

\author{
R. SHARMA ${ }^{*}$, W. REN ${ }^{* *}$, S. A. MCDONALD ${ }^{\dagger}$ AND Z. YANG ${ }^{\dagger \dagger}$ \\ Indian Institute of Technology Mandi (IIT) \\ Mandi, India, 175005 \\ e-mail: rajnishsharma@iitmandi.ac.in \\ * Northwest A\&F University, \\ Yangling, China, 712100 \\ e-mail: wenyuange304@nwsuaf.edu.cn \\ ${ }^{\dagger}$ University of Manchester, \\ Manchester, UK \\ e-mail: sam.mcdonald@manchester.ac.uk \\ ${ }^{\dagger}$ Coventry University, \\ Coventry, UK \\ e-mail: ac1098@ coventry.ac.uk
}

Key words: Concrete Fracture, In-situ XCT Testing, Damage Propagation, Microstructure Reconstruction

\begin{abstract}
A meso scale compression test of the concrete specimen has been conducted in an in-situ X-ray computed tomography (XCT) facility. Concrete contained coarse aggregates of $5 \mathrm{~mm}$ and normal Portland cement. A specimen of $20 \times 20 \times 20 \mathrm{~mm}^{3}$ size was studied under three cycles in compression. It has been scanned after the completion of each stage in a cyclic (e.g. immediate after loading to the peak and after the unloading) and two dimensional (2D) images of the microstructure were recorded with a resolution of $16.5 \mu \mathrm{m}$. A great amount of pre-existing damage in the form of micro cracks and voids has been revealed in the scanned images. Further, the micro-mechanisms, such as crack opening and closing, crack tip extension and diversion, crack tip blunting, and elastic recovery of the crack openings and closures were also observed in the images from different cycles. Finally, digital volume correlation (DVC) was utilized to get the macro displacement fields during each load cycle.
\end{abstract}

\section{INTRODUCTION}

Quasi-brittle failure of concrete is a very complex phenomenon, due to its complicated multi-phasic microstructure consisting of randomly distributed aggregates, mortar and voids of different shapes, sizes and volume fractions. The macroscopic overall failure of concrete depends on the microscopic properties and localised failure characteristics of its constituents. The experimental investigation of the essentially threedimensional (3D) micro-scale failure has been very challenging, due to the limitations of conventional techniques, which are mostly 2D surface based (e.g., photoelastic methods) or destructive post-mortem analyses. 
An increasingly popular technique, capable of scanning the 3D microstructures and observing the micro-scale failure process under progressive loading, is the in-situ X-ray computed tomography (XCT). Its main advantages include the non-destructive nature, multi-length scale resolutions and wide applicability to many solid materials [1]. Most of the existing XCT studies are restricted to the reconstruction of $3 \mathrm{D}$ internal microstructures of various materials [2-6] without loading. Recently, some in-situ XCT tests, by integrating a loading rig during the scan were carried out to investigate the internal 3D damage and fracture evolution in some materials, examples Limodin et al. [7] in nodular graphite cast iron, Bay et al [8] in bones, Brault et al. [9] in laminated composites, and Mostafavi et. al [10] in nuclear graphite, and Patterson et. al [11] in aluminium foam.

Limited in-situ XCT tests were also found for concrete-like quasi-brittle materials. For example, Landis and Nagy [12] carried out insitu compressive XCT tests on cylindrical mortar specimens of size $4 \mathrm{~mm}$ and investigated the crack initiation and growth in the post-peak regime. Wang et al. [13] established a relation between the overall damage and representative defect area using $\mathrm{XCT}$ reconstructed images of asphalt concrete. Wong and Chau [14] related the nucleation of voids to the gray scale value of pixels in normal and high performance concrete under various compressive loadings. Wan and Xue [15] conducted in-situ XCT compression tests on cement and developed relations between the average gray scale value and the damage by comparison of scanned images. Yang and co-workers $[16,17,18]$ have studied the $40 \mathrm{~mm}$ concrete cubes under in-situ XCT compression tests. They developed the $2 \mathrm{D}$ and $3 \mathrm{D}$ finite element models based on reconstructed images for fracture modelling of the concrete. Most of these limited number of in-situ XCT tests were focused on the overall estimation of the damage variable, whereas the localised failure characteristics are rarely investigated. In addition, most of them have applied monotonic loading schemes and in-situ XCT tests under cyclic loading have not been reported to the best knowledge of the authors.

In this study, an in-situ micro XCT test of a concrete cube under cyclic compressive loading was carried out, as an attempt for better understanding of the different failure mechanisms using image analysis. Also, recently-developed digital volume correlation (DVC) technique $[8,19,20]$ is utilised to calculate the full 3D deformation fields for the different loading.

\section{IN-SITU XCT TEST}

A small concrete specimen of size nearly $20 \times 20 \times 20 \mathrm{~mm}^{3}$ was cut from a $40 \times 40 \times 40 \mathrm{~mm}^{3}$ concrete cube. The concrete was same as used earlier by authors [17] and made of $5 \mathrm{~mm}$ aggregate size and ordinary Portland cement. The objective of the small size of the specimen was to get an enhanced resolution and good quality of finite element mesh for the future fracture simulation. In past, the $40 \mathrm{~mm}$ size of the specimen was scanned [17] and it was rather difficult to model the same specimen in finite element analysis. Therefore, the specimen size was reduced to enhance the resolution and the total numbers of degrees of freedom of FE mesh. Manchester X-ray imaging facility in the school of materials at University of Manchester, UK, was utilized for the scans. Tungsten target was used with an accelerating voltage of $100 \mathrm{kV}$ and $200 \mu \mathrm{A}$ current for all the scans. Flat X-ray attenuations were obtained and a black reference was made before starting the experiment. The projection images were obtained through a silicon flat detector with an exposure time of $1.4 \mathrm{~s}$. Nearly, 1500 projection images have been captured by rotating the stage through 360 degree. These radiographs were then converted in to $3 \mathrm{D}$ material image using standard filter back reconstruction algorithm. For each scan, the corrections were made to reduce the effect of the artifacts like beam hardening and ring effect. Finally, the satisfactory contrast for reconstructed 2D XCT images was obtained with a resolution of 16.5 $\mu \mathrm{m}$. 3D images of all scans have been visualized using commercial software $[21,22]$. 

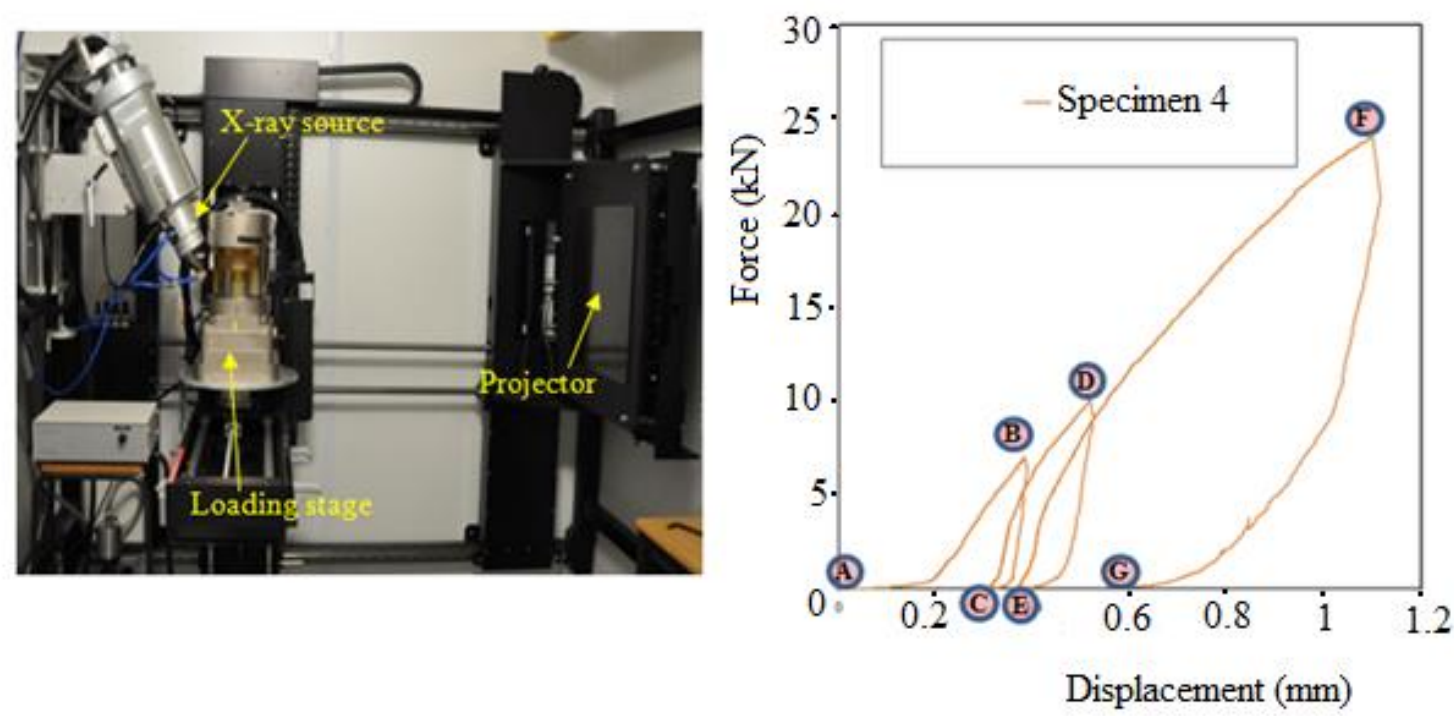

Figure 1: a) In-situ experimental setup, b) Load displacement curve.

The contrast in these images was further enhanced by removing the noise using mean and median filters. A complete test setup is shown in the Fig.1a.

Load was applied using a loading stage (Deben rig) with a speed of $0.5 \mathrm{~mm}$ per $\mathrm{min}$ in three cycles with load control. The loaddisplacement curve obtained is shown in Fig.1b. In the first cycle, the specimen was loaded up to $7 \mathrm{KN}$ and then unloaded to $50 \mathrm{~N}$. Three scans were made during this cycle. First scan was taken at position A (without any load), 2nd scan at position B (at $7 \mathrm{KN}$ ) and 3rd scan at position $\mathrm{C}$ as marked in Fig.1b. In the 2nd cycle, the load was increased up to $10 \mathrm{kN}$ and again unloaded to the $50 \mathrm{~N}$. 4th and 5th scans were made accordingly at the loaded and unloaded positions (ref. $\mathrm{D}$ and $\mathrm{E}$ points in Fig.1b). In 3rd cycle, the load was applied up to $24 \mathrm{kN}$ and again unloaded back to the $50 \mathrm{kN}$ and consecutive scans were named as $F$ and $G$ respectively as shown in the Fig.1b.

Fracture process under the loading history has been analyzed with respect to overall damage propagation in the specimen during the loading history. The details of the crack propagation from reconstructed XCT images are discussed in the next section.

\section{OBSERVATION ON DAMAGE PROPAGATION}

Four 2D slices (from each scan A, B, D and $F)$, which represents the same location in the specimen under different loads are used to describe the damage propagation process as shown in Fig.2.

It is clear from the Fig.2a that the specimen contained some pre-existing interfacial, surface and internal cement cracks along with voids. There are many micro pores (referred as small pores in Fig.2) present on the aggregate/cement interface and also within the cement phase. Big voids, which are relatively very large as compared to micro-pores, are also identified in the concrete images (referred as big pores in Fig.2). The initial cracks present in the concrete may be either formed during the hardening process or originated due to the specimen preparation process. Some other researchers have also discussed the presence of the initial cracks in concrete $[6,12]$. Some of the pre-existing cracks and micro-voids have been closed due to the initial application of compressive load as shown in scan B (refer red circle in Fig.2a\&b). 

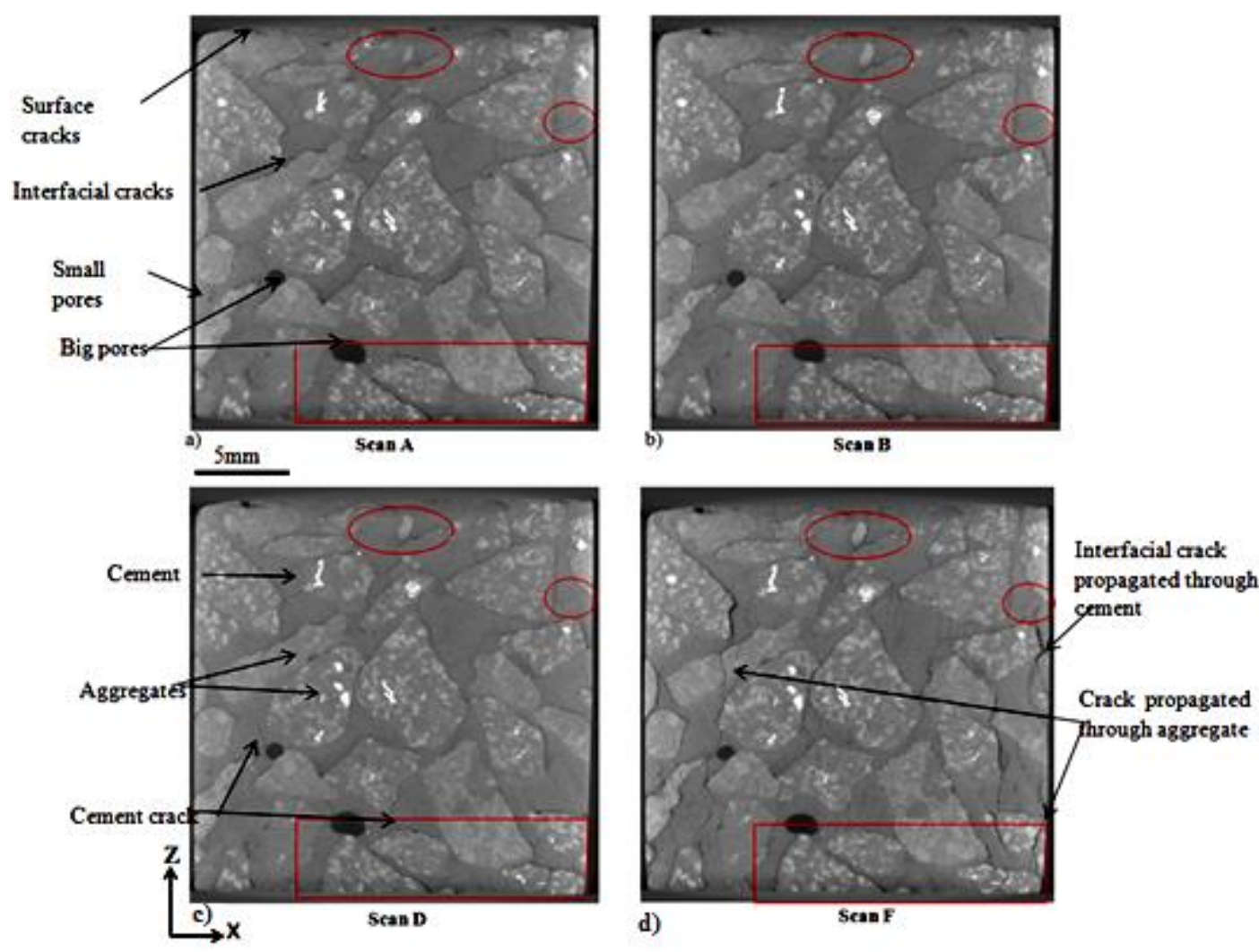

Figure 2: Damage description in reconstructed 2D slices from different loading stages.

It is also clear from Fig.2b that some aggregate readjustments near the boundaries have also taken place in the specimen during the initial loading. The crack closures are increased with further increments of the load. Many cracks parallel to the loading direction have been appeared in the image obtained from the scan F (see Fig.2d). This means that the cracks network has sufficiently developed all over the specimen to reach the failure. The overall crack propagation is a very complex phenomenon and is difficult to understand from 2D images alone. To develop a good understanding of the fracture process, the crack network has been segmented for all the scans. 3D images of the reconstructed cracks network for different loading stages have been shown in Fig.3.

During the reconstruction, a compromise has been made to make the network image clearer for visualization. The tinny individual cracks less than 100 voxels have been filtered out. Fig.3a shows pre-existing 3D network of the cracks and voids in the specimen. A 3D view has given the brief description of the distribution of pre-existing cracks. As one can see the crack is not a flat and smooth surface. It is a combination of many uneven surfaces and sometime connected with very small bridging cracks. One can compare closely Fig.3a\&b to observe some crack closures due to initial compressive loading. There are very small changes in the configuration of the cracks network in these images. Hence, the stage can be considered as the part of the elastic loading. Fig. $3 \mathrm{c}$ has clearly revealed that there is a substantial increase in the micro crack density under $10 \mathrm{kN}$ load. Many small cracks have been originated at different places and many cracks have been bridged the preexisting network. This kind of detailed information for the cracks network cannot be concluded from 2D slices alone. Final macro crack surface under load of $24 \mathrm{kN}$ has been shown from scan F in Fig.3d. Many new crack surfaces, mostly parallel to the loading direction have bridged to form a wider uneven complex crack network. 


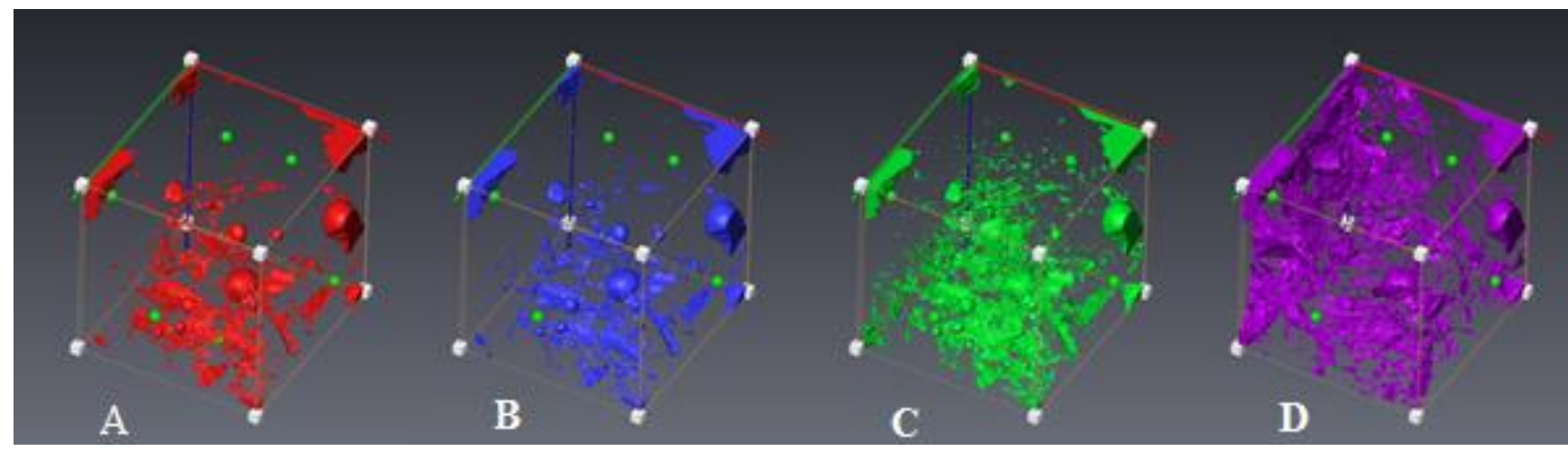

Figure 3: Reconstructed cracks network of specimen.

This section for overall damage can be concluded as follow: some of the pre-existing cracks closed during the initial loading, then the cracks initiated parallel to the loading direction at distinct places, which were bridged to form a macro cracks network due further application of load. Next section describes the localized distribution of strain in the specimens by using the digital volume correlation.

\section{DIGITAL VOLUME CORRELATION}

Digital volume correlation (DVC) is a 3D extension of the well-known digital image correlation (DIC) technique used in 2D. In DIC, two images (one from the original and other from deformed configurations) were divided into small subsets of areas and then correlated to find out the deformation gradient of these subsets. In the similar way, in DVC the total volume had been divided into small sub-volumes which contain some point of interest. These points of interest had been tracked in the sub-volumes of deformed image. By comparing two sub-volumes from deformed and undeformed configurations, the displacements were interpolated for each subvolume. Before performing the correlation on the volumes, these images were register to reduce the rigid body movement effects. The volumes were manually translated in three directions to obtain the accuracy of nearly one or two pixels for the registration. Final processed images were cropped and saved in raw format. These raw images were used in the DaVis (a Commercial software package
[9]) to correlate the specimen volumes from different cycles. The correlation algorithm is based on the fast Fourier transform. One can refer the pioneer work of Bay et.al [8] for more details on this technique. Three window sizes as $128 \times 128 \times 128,64 \times 64 \times 64$ and $32 \times 32 \times 32$ voxels were investigated for the optimum results. Two passes have been made with an overlap of $50 \%$ to achieve the results. Fig.4 shows the variation of the $z$-direction displacement $(w)$ on the center slice of $\mathrm{Xz}$ plane under $7 \mathrm{kN}$ load. Here, $z$ is the loading direction. Figs.4a, b and c show the contours of $w$ for window size 128, 64 and 32 respectively. The displacement contours are smoother as the size of the window is reduced. To show the variation of the $w$ displacement in the loading direction (thickness direction), the displacements $(w)$ has been average over each slice in xy plane. Fig. 5 shows the variation of the $w$ in the thickness direction of the specimen and the effect of the window size. In Fig.5, $w$ has increased up to the $1 / 5$ th of the specimen height and then linearly decreased toward the bottom. Near the bottom it became as zero and then negative. Similar trend obtained by the three window sizes. The magnitude of the displacement has slightly increased with the reduction in the window size. The predicted magnitude of the displacements by window sizes 64 and 32 are very near to each other. Hence, one can conclude that the 32 is sufficiently large to correlate the displacement gradients and enough small to capture the local variations. Note that all these local variations are not the representations of the micro-strains, these are 
macroscopically local variations.

These can be treated as the representations of the heterogeneity at the macro level. Due to the heterogeneity and presence of the defects the displacements are increasing first and negative at the bottom of the specimen. One can also notice that the neutral plane has also got shifted towards the middle of specimen due to the application of the load.
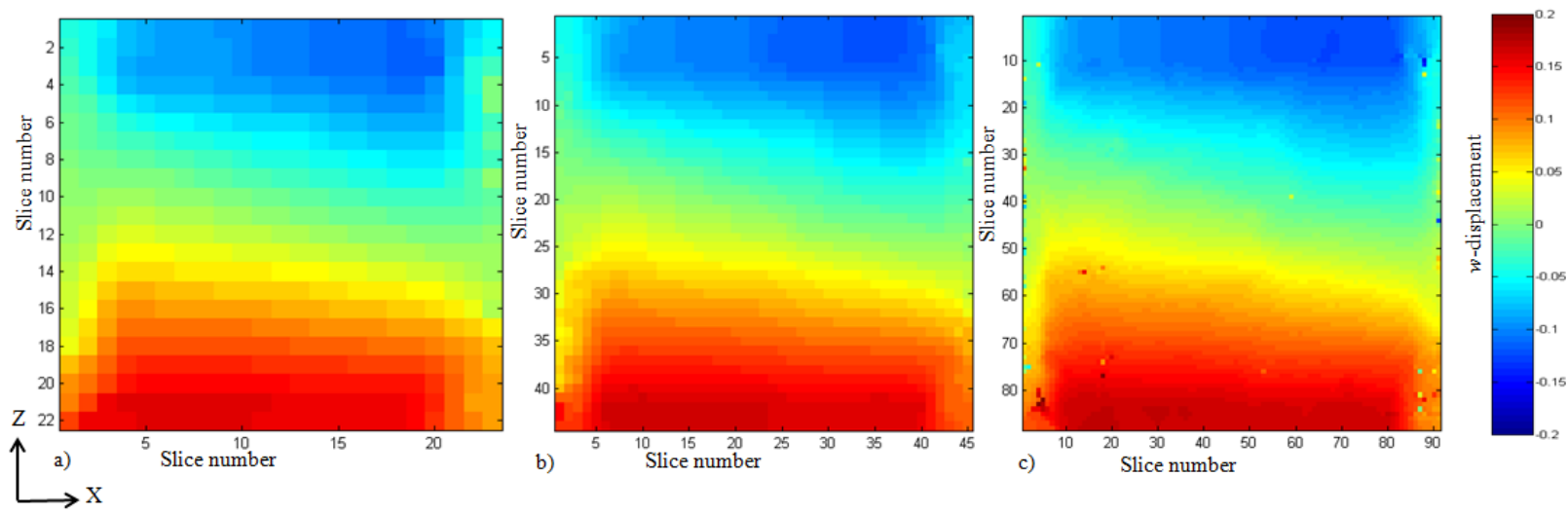

Figure 4: Effect of the window size selection on the displacements.
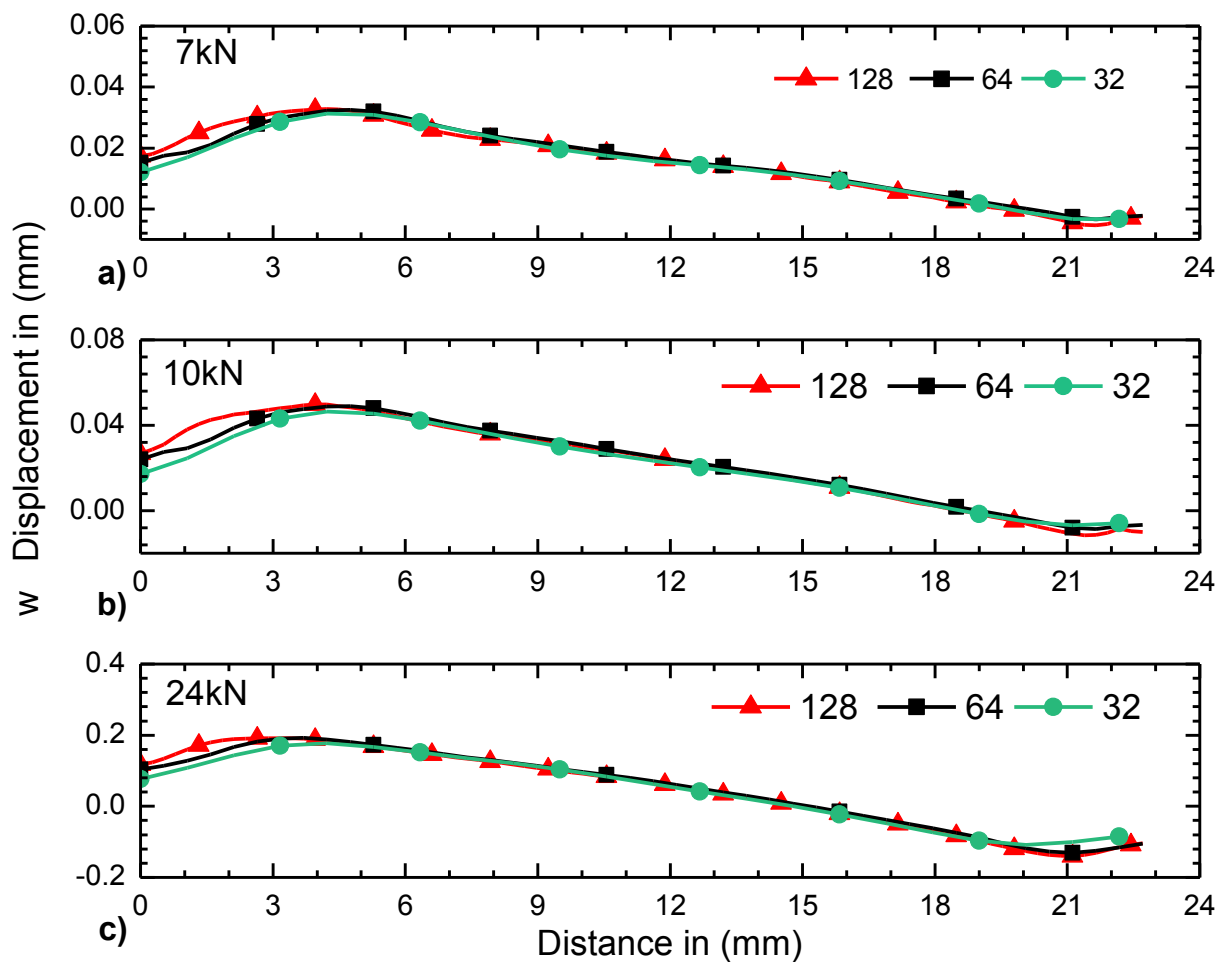

Figure 5: variation of the $\mathrm{w}$ displacement along the thickness of the specimen. 

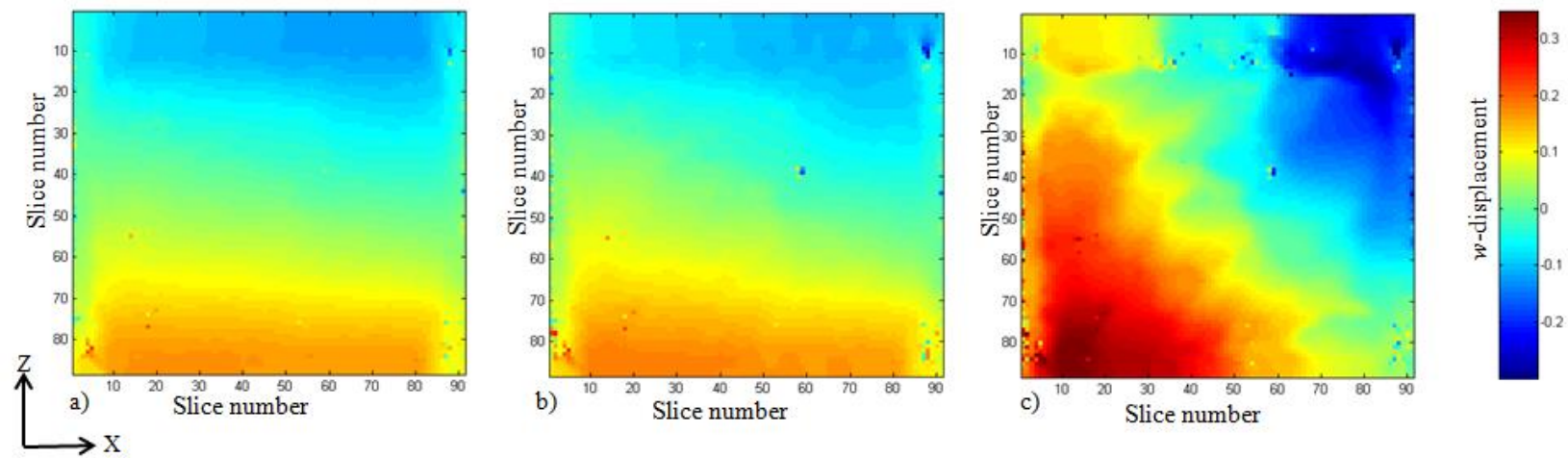

Figure 6: variation of the $w$ displacement along the thickness of the specimen.

More light on the variation of the deformations can be thrown with the contours of the w-displacements. Fig. 6 shows the deformation contours under peak loads of the cycles. There is an increase in the positive displacements at the bottom of the image (ref. Figs.6a and b), whereas the negative displacements i.e movement from the top to bottom of the image is reduced. The contours have also slightly rotated clockwise. With the application of the next loading, displacements are concentrated at the both ends and produced a zero plane nearly at the 45 degrees.

\section{CONCLUSIONS}

In-situ cyclic compression test has been conducted on a small scale specimen of concrete to explore the damage in the pre-peak region of load displacement curve. It is revealed from the XCT images that specimen contained initial inherent internal defects in addition to surface damage. The local fracture mechanisms such as crack opening and closing during the loading and unloading history have been revealed through the reconstructed XCT images. Elastic recovery of the crack openings and closures has also been observed. 3D image has revealed that macro crack has an irregular shape and contained many micro fronts liable to extension. Also there are many micro cracks originated before the appearance of macro crack. Next, the DVC has been used to find out the localized macro displacements in the specimen. The distribution has revealed the specimen heterogeneity. The displacement contours are highly heterogeneous in the specimen near the peak load. The average $w$ displacements over xy plane are observed high nearly at a distance of $5 \mathrm{~mm}$ from the loading edge. This distance is equal to the average distance of the aggregate.

\section{ACKNOWLEDGEMENT}

The research is funded by UK EPSRC (No. EP/J019763/1), Manchester X-ray Imaging Facility was funded in part by the EPSRC (No. EP/F007906/1).

\section{REFERENCES}

[1].Landis EN, Keane DT. X-ray microtomography. Mat Charact 2010; 61(12): 1305-1316.

[2].Magne P, Efficient 3D finite element analysis of dental restorative procedures using micro-CT data. Dent Mats 2007; 23: 539-548.

[3].Ulrich D, Rietbergen BV, Weinans H, Rüegsegger P. Finite element analysis of trabecular bone structure: a comparison of image-based meshing techniques. J. of Biomech 1998; 31: 1187-1192.

[4].Coindreau O, Vignoles G, Cloetens P. Direct 3D microscale imaging of carboncarbon composites with computed holotomography. Nucl Inst and Meth in Phy Res Sec B: Beam Inter with Mat and Atoms 2003; 200: 308-314.

[5].Sharma R, Deshpande VV, Bhagat AR, Mahajan P, Mittal RK. X-ray tomographical observations of cracks and voids in 3D carbon/carbon composites. Carbon 2013; 60: 335-345.

[6].Masad E, Muhunthan B, Shashidhar N, Harman T. Internal Structure 
Characterization of Asphalt Concrete Using Image Analysis. J. Comput. Civ. Eng. 1999; 13(2): 88-95.

[7].Limodin N, Réthoré J, Buffière JY, Hild F, Roux S, Ludwig W, Rannou J, Gravouil A, Influence of closure on the 3D propagation of fatigue cracks in a nodular cast iron investigated by X-ray tomography and 3D volume correlation, Acta Mat. 2010; 58(8):2957-2967.

[8].Bay BK, Smith TS, Fyhrie DP, Saad M. Digital Volume Correlation: Threedimensional Strain Mapping Using X-ray Tomography. Exp Mech 1999; 39(3):217226.

[9]. Brault R., Germaneau A, Dupré JC, Doumalin P, Mistou S, Fazzini M. In-situ Analysis of Laminated Composite Materials by X-ray Micro-Computed Tomography and Digital Volume Correlation. Exp Mech 2013; 53:11431151

[10]. Mostafavi M, Baimpas N, Tarleton E, Atwood RC, McDonald SA, Korsunsky AM, Marrow TJ. Three-dimensional crack observation, quantification and simulation in a quasi-brittle material, Acta Mat 2013; 61(16): 6276-6289.

[11]. Patterson BM, Henderson K, Smith Z. Measure of morphological and performance properties in polymeric silicone foams by X-ray tomography, J. of Mat Scie 2013; 48(5): 1986-1996.

[12]. Landis EN, Nagy EN. Threedimensional work of fracture for mortar in compression, Engg Frac Mech 2000; 65:223-234.

[13]. Wang LB, Frost JD, Voyiadjis GZ, Harman TP. Quantification of damage parameters using X-ray tomography images. Mech of Mat 2003; 35(8): 777790.

[14]. Wong RCK, Chau KT. Estimation of air void and aggregate spatial distributions in concrete under uniaxial compression using computer tomography scanning. Cem and Conc Res 2005; 35 (8): 15661576.

[15]. Wan K, Xue X. In situ compressive damage of cement paste characterized by lab source X-ray computer tomography. Mat Chara 2013; 82: 32-40.

[16]. Huang, Y.J., Yang, Z.J, Ren, W.Y., Liu, G.H., Zhang, C.Z., 2015 3D In-situ XCT Image Based Meso-scale Fracture Modelling and Validation of Concrete Using Voxel Hexahedron Meshing and Damage Plasticity Model. International Journal of Solids and Structures 67-68, 340-352.

[17]. Yang, Z.J., Ren, W.Y., Mostafavi, M., Mcdonald, S.A., Marrow, T.J., 2013. Characterisation of 3D fracture evolution in concrete using in-situ X-ray computed tomography testing and digital volume correlation. In: VIII International Conference on Fracture Mechanics of Concrete and Concrete Structures. Toledo, Spain, CIMNE.

[18]. Ren W, Yang Z, Sharma R, Zhang Ch, Withers PJ. Two-dimensional X-ray CT image based meso-scale fracture modelling of concrete. Engg Frac Mech 2015; 133: 24-39.

[19]. Pierron F, McDonald SA, Hollis D, Fu J, Withers PJ, Alderson A. Comparison of the Mechanical Behaviour of Standard and Auxetic Foams by X-ray Computed Tomography and Digital Volume Correlation. Strain 2013; 49:467-482.

[20]. Davis, digital volume correlation (DVC) package, www.lavision.de.

[21]. Avizo, 3D image processing package, http://www.fei.com/software/avizo3d/

[22]. Image J, Image processing package, http://imagej.nih.gov/ij/index.html

[23]. Lemaitre J. A Course on Damage Mechanics, Springer Berlin Heidelberg 1991, DOI 10.1007/978-3-642-18255-6 